\title{
XMM OBSERVATIONS OF POLARS FROM THE SDSS
}

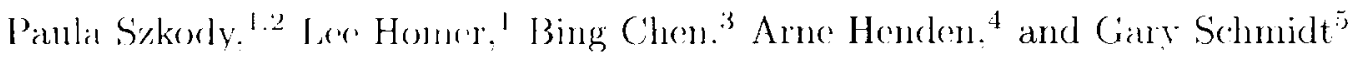 \\ RESTIVIS
}

Hemos llevale a cabo observariomes XMM/ ie is polares débiles descubiertas por el Sloan Digital Sky Survey.

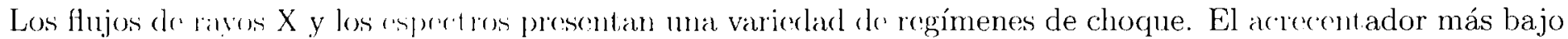
posee propiedates de raves X ronsistrules en un origen on la estrella secundaria. Las curvas de luz de todos

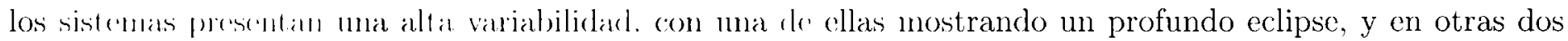
con crielencias de variakiones melarionadas conl la órbita.

\section{ABSTRRAC'I}

We conducted XM/N olswivalions of 5 faint Polars discovered by the Sloan Digital Sky Survey. The X-ray fluxes and spectra show a variety of shock regines. The lowest accotor has X-ray properties consistent with an origin on the secendary star. The light curves of all nistems show large variability, with one showing a deep ecliphe. and with sone cviclence for orbital-related variations in '2 others.

\section{kill Wirts: BINARIES: CLOSE X-RAYS: BINARIES}

\section{INTTROD)U(ZTIO)N}

The first 2 years of the Siloatn Digital Sky Survey (SDSS) hatre revealed a variety of cataclysinic variables (Sykody et al. 20(0): 20):3a.b), anong them soreral new systoms containing highly magnetic white dwarfis (known as Polars or AN Her strateme). Due to the faint magnitudes reached by the SDSS, the new systems include some of the the lowest masstransfer rate sistemes known. For Polars, this means that many objects may be in the regime where the alectrons cool primarily by cretolron emission or where the shock degenerates into a bombardnent solution (sere IV ickramasinghe \& Forrario (2000) for a description of these (ansess). To gain a better menderstanding of the shock regimes and areretion char-

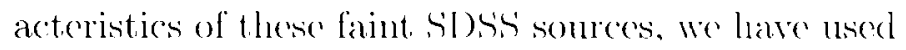
the high sensitivity and good energy coverage of the TMM satellite fo observe 5 of these new sourees. The characteristirs of the 5 Polin's are summarized in the Table below and a brief smmmary of the results follows. For berity. we abbreviate the someres to their first few digits in RA but the full source identifications are in stokody et al. (20)22, 2003a.b).

\section{SLSS.J $1553+55$}

This SDSS soure is one of two that were found to have extrene arelotron harmonies (Sskody et

\footnotetext{
${ }^{1}$ Dept. of Astronomy l Washingum, lsil.

${ }^{2}$ Dept. of Physics d Istronomy ast I Isi

${ }^{3}$ VILSP'A. Spain.

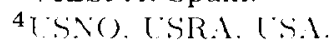

${ }^{5}$ Steward Observatory. L Arimana. ISi.
}

TABLE 1

XMIM OBSERVATIONS OF POLARS

\begin{tabular}{lrccrr}
\hline SDSSJ & $\mathrm{P}(\mathrm{m})$ & $\mathrm{V}(\mathrm{mag})$ & UT Date & PN $(\mathrm{c} / \mathrm{s})$ & $(\mathrm{ks})$ \\
\hline $0155+00$ & 87 & 15.6 & $7-04-03$ & $0-0.05$ & 11.1 \\
$0729+36$ & 120 & 20.6 & $10-31-02$ & $0-0.08$ & 7.5 \\
$0752+36$ & 162 & 19.0 & $10-31-02$ & $0.4-1.1$ & 7.4 \\
$15.53+5+5.5$ & 263 & 17.6 & $2-17-03$ & $0.01-0.04$ & 10.4 \\
$1.553+5+5.5$ & 263 & 17.6 & $3-12-03$ & $0.02-0.06$ & 9.7 \\
$1700+40$ & 115 & 18.0 & $8-11-03$ & $0-0.25$ & 9.0 \\
\hline
\end{tabular}

al. 20013a) that are highly modulated in the optical throughout their orbital period $(4.4 \mathrm{hr}$ for SDSSJ1553) as the viewing angle of the magnetic pole changes. Fitting these harmonics indicated that SDSSJ1553 had a $60 \mathrm{NG}$ field strength with a very low shock temperature $(<1 \mathrm{keV})$ and an extremely low specific accretion rate $\left(10^{-14} \mathrm{M} . / \mathrm{yr}\right)$, placing it in the bombardment accretion regime. $X M M$ data is consistent with this picture, as the PN count rate is very low and there is no periodic variation evident in either of the 2 observations. The $\mathrm{X}$-ray flux that is observed all comes from energies below $2 \mathrm{keV}$ and the best fit to the $0.2-10 \mathrm{keV}$ spectra of both observations is with a $188 \mathrm{el}$ bremsstrahlung source. As the distance to SDSS15.53 is known to be about 100pe (from the identification of $\mathrm{TiO}$ bands of an $\mathrm{M} 5 \mathrm{~V}$ star evident in the near-IR), the X-ray luminosity comes out to be about $10^{30} \mathrm{ergs} / \mathrm{s}$. The flux, light curve and spectrum are all consistent with an origin from the secondary star. rather than from active accretion at 
a magnetic pole. A previous $S A X$ observation of AM Her during a low state had identified the X-rays as possibly having a large contribution from the activity level of the secondary, but the spectrum was too hard $(>3.6 \mathrm{keV})$ to be attributed solely to a coronal source (deMartino et al. 1998). Thus, SDSSJ 1553 may provide the first good evidence of the activity level of the secondary in a cataclysmic variable.

\section{SDSSJ0155}

The first optical observations of this bright SDSS Polar revealed deep eclipses on a period of $87 \mathrm{~min}$ (Szkody et al. 2002). The $X M M$ data cover more than 2 orbital periods and show deep eclipses in Xrays at the same phases as the optical eclipses observed preceding and following the X-ray observations. The X-ray spectrum can be fit with both hard (14 keV bremsstrahlung) and soft (125 eV black body) components. The soft component is harder than typically found in Polars, but this may be the result of calibration problems with the $X M M \mathrm{PN}$ and MOS detectors below $0.5 \mathrm{keV}$.

\section{SDSSJ 1700}

Optical analysis of this polar revealed a $30 \mathrm{MG}$ field strength and a low inclination so that one accretion pole is viewed throughout the $115 \mathrm{~min}$ orbit (Szkody et al. 2003b). While the optical light curve shows a strong 2.5 mag periodic modulation throughout its orbit, the $X M M$ PN light curve shows strong variability for the first few ks which does not repeat on the orbital timescale. The spectrum can be fit with a soft black-body and bremsstrahlung components, although the fit is not well-constrained. Transient flaring-like activity is common in Polars and has been observed in both active and low states of AM Her and UZ For (deMartino et al. 1998; Pandel \& Cordova 2002). Thus, it appears that the variability in the mass transfer dominates the orbital variability at the time of the $X M M$ observation of SDSSJ1700.

\section{SDSSJ0752}

Optical photometry shows a 1.5 mag modulation of this system on its $2.7 \mathrm{hr}$ period, typical of the changing view of a single accretion pole. The $X M M$ data only cover about 0.7 phase. There is a narrow eclipse-like feature near the start of the MOS observations, which unfortunately is not covered in the PN observation which started later. This feature looks very much like an X-ray eclipse, and it occurs at the same phase as the minima of the optical (obtained 11 hrs later), although it is much narrower than the optical minimum. However, without coverage of several complete orbits, it is impossible to tell if this is sporadic or periodic behavior. The X-ray spectrum is best fit with a combination of a $97 \mathrm{cV}$ black-body and a $20 \mathrm{keV}$ bremsstrahlung component.

\section{SDSSJ0729}

This optical (and X-ray) faint system also shows a one-mag variation in the optical throughout its 2.6 hr orbit. The XMM observation covered $80 \%$ of an orbit. During the first part ( 0.55 in phase), the Xrays are close to zero, but then there is a large rise in count rate for the last $2 \mathrm{ks}$ of the observation. As in the case of SDSSJ0752, we will need further data to determine if this rise is sporadic or associated with the emergence of the X-ray emitting pole from selfeclipse. In this case, there is no contemporaneous ground coverage and the Optical Nonitor data have too low $\mathrm{S} / \mathrm{N}$ to provide much insight into the transient accretion.

\section{CONCLUSIONS}

$X M M$ is a good instrument for detecting the faint $\mathrm{X}$-ray emission of low accretion rate Polars found in SDSS. The X-rays detected from the lowest accretor, SDSSJ1553 are consistent with an origin from an active late $M$ secondary star, rather than an accretion shock. Thus, these lowest accretors can be used to probe the activity level of the secondaries in fast-rotating, short period binaries. The X-ray light curves of the other four all show large variability, although only SDSS0155 is clearly periodic, with a deep, narrow X-ray eclipse coincident with the optical eclipse. SDSSJ0752 also shows what may be an X-ray eclipse feature. SDSSJ1700 shows evidence for transient accretion variability. Except for SDSSJ1553, the sources can be fit with both a soft and hard component, indicating that there is an accretion shock which emits hard X-ray and heats the underlying white dwarf.

This work was supported by NASA grant NAG512938 and NSF grant AST-02-05875 to the University of Washington and NSF grant AST 97-30792 to Steward Observatory.

\section{REFERENCES}

deMartino, D. et al. 1998, AAp, 333, L31

Pandel, D. \& Cordova, F. A., 2002, MNRAS, 336, 1049

Szkody, P. et al. 2002, AJ, 123, 430

Szkody, P. et al. 2003a, ApJ, 583, 902

Szkody, P. et al. 2003b, AJ, 126, 1499

Wickramasinghe, D., \& Ferrario, L. 2000, PASP. 112. $\$ 13$ 